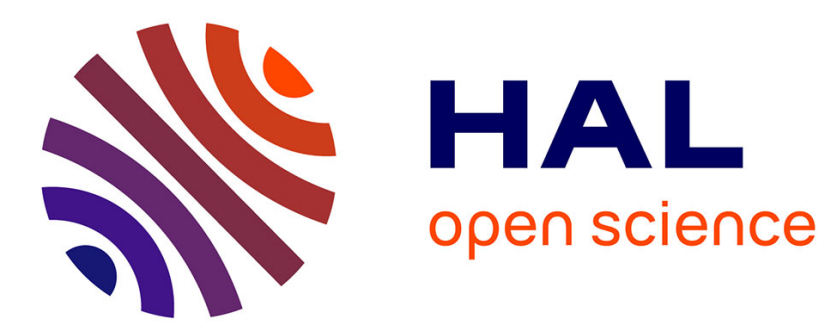

\title{
Domain decomposition method and elastic multistructures : the stiffened plate problem
}

Frédéric d'Hennezel

\section{To cite this version:}

Frédéric d'Hennezel. Domain decomposition method and elastic multistructures: the stiffened plate problem. [Research Report] RR-1800, INRIA. 1992. inria-00074874

\section{HAL Id: inria-00074874 \\ https://hal.inria.fr/inria-00074874}

Submitted on 24 May 2006

HAL is a multi-disciplinary open access archive for the deposit and dissemination of scientific research documents, whether they are published or not. The documents may come from teaching and research institutions in France or abroad, or from public or private research centers.
L'archive ouverte pluridisciplinaire HAL, est destinée au dépôt et à la diffusion de documents scientifiques de niveau recherche, publiés ou non, émanant des établissements d'enseignement et de recherche français ou étrangers, des laboratoires publics ou privés. 


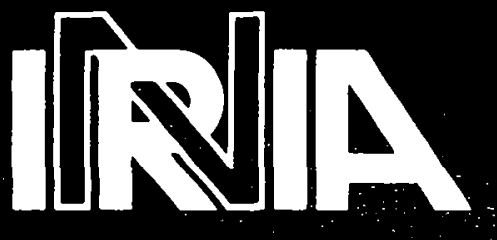

UNITÉ DE RECHERCHE URIA-ROCOUENCOURT

\section{Rapports de Recherche}

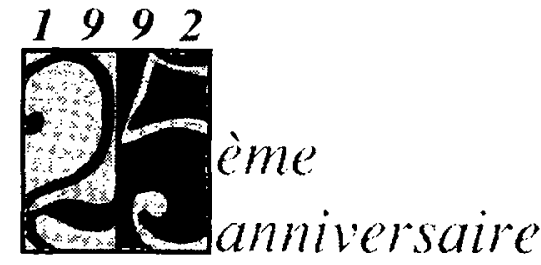

$\mathrm{N}^{\circ} 1800$

\section{Programme 6}

Calcul Scientifique, Modélisation et

Logiciels numériques

\section{DOMAIN DECOMPOSITION} METHOD AND ELASTIC MULTISTRUCTURES :

THE STIFFENED PLATE PROBLEM
Institut National de Recherche en informatique eten Automátique

Domaine de Voluceat

Rocquencourt

$$
\text { BP105 }
$$

78153 Le Chesnay eedex

$$
\text { France. }
$$

Tél:(1) 39635511
Frédéric D'HENNEZEL

Novembre 1992 


\title{
Domain Decomposition Method and Elastic Multistructures : the Stiffened Plate Problem.
}

\author{
Frédéric d'Hennezel
}

\begin{abstract}
Domain decomposition methods allow faster solution of partial differential equations in many cases. The efficiency of these methods mainly depends on the variables and operators chosen for the coupling between the subdomains; it is the preconditioning problem. In the modeling of multistructures, the partial differential equations have some specific properties that must be taken into account in a domain decompsition method. Different kinds of elliptic problems modeling stiffened plates in linearized elasticity are compared. One of them is remarkable as far as domain decomposition is concerned, since it is possible to associate particularly efficient preconditioner. A theoretical estimate for the conditioning is given, which is confirmed by several numerical experiments.
\end{abstract}

\section{Méthode de Décomposition de Domaine et Multistructures Elastiques : les Plaques munies de raidisseurs.}

\author{
Frédéric d'Hennezel
}

Résumé. Les méthodes de décomposition de domaine permettent, dans de nombreux cas, de résoudre plus rapidement des équations aux dérivées partielles. L'efficacité de ces méthodes dépend pour l'essentiel du choix des variables et des opérateurs traduisant le couplage entre les sous-domaines ; c'est le problème du préconditionnement. Dans la modélisation des multistructures, les équations aux dérivées partielles présentent certaines propriétés spécifiques à prendre en compte dans une méthode de décomposition de domaine. Différents types de problèmes elliptiques modélisant les plaques raidies en élasticité linéarisée sont comparés. L'un d'eux s'avère remarquable du point de vue de la décomposition de domaine, puisqu'il va être possible d'y associer un préconditionneur particulièrement efficace. Une estimation théorique du conditionnement est donnée, appuyée par plusieurs expériences numériques.

0 A paraître dans "Numerische Mathematik" Springer Verlag. 


\title{
Domain Decomposition Method and Elastic Multistructures : the Stiffened Plate Problem
}

\author{
Frédéric d'Hennezel \\ I.N.R.I.A. \\ Domaine de Voluceau, Rocquencourt, \\ B.P. 105, 78153 Le Chesnay Cedex, France.
}

September 30, 1992

\begin{abstract}
Domain decomposition methods allow faster solution of partial differential equations in many cases. The efficiency of these methods mainly depends on the variables and operators chosen for the coupling between the subdomains; it is the preconditioning problem. In the modeling of multistructures, the partial differential equations have some specific properties that must be taken into account in a domain decompsition method. Different kinds of elliptic problems modeling stiffened plates in linearized elasticity are compared. One of them is remarkable as far as domain decomposition is concerned, since it is possible to associate particularly efficient preconditioner. A theoretical estimate for the conditioning is given, which is confirmed by several numerical experiments.
\end{abstract}

\section{Introduction}

A multistructure is a multidimensional assembly of beams, plates, shells or threedimensional structures, attached together with junctions. The aim of this paper is to introduce a specific domain decomposition method for the numerical problem associated with a multistructure : the elastic stiffened plate.

Before applying a numerical algorithm to a discretized partial differential equation, it is necessary to justify the mathematical modeling of the problem. We compare two different approachs of multistructure modeling.

The fisrt one uses known equations of beams, plates, or shells. Using some hypothesis on the behavior of the junction, we obtain new equations with a coupling between the different substructures. For example Bernadou, Fayolle \& Léné [1989] consider the modelization of the junction between plates with arbitrary angle, Bernadou [1989] the junction between shells, and Janovsky \& Prochăska $[1976,1978]$ the case of stiffened plates (which can be seen as a junction between a plate and beams). In the above 
mentioned papers, the hypothesis that determine the behavior of the junction are of mechanical nature and have not been justified from an asymptotic analysis.

The paper by Ciarlet, Le Dret \& Nzengwa [1989] opened a fruitful way for the mathematical justification in junction modeling. Their paper investigates the case of a junction between a plate and a three-dimensional structure. The justification is done from the three-dimensional elasticity problem set in the whole structure. As the thickness of the plate tends to zero, a new multi-dimensional 3D-2D problem is obtained. In the $2 \mathrm{D}$ part we have classical plate equations, coupled in the $3 \mathrm{D}$ part with the system of three-dimensional elasticity. This coupling results of the asymptotic study of the junction conditions as the thickness of the plate tends to zero. Other kinds of multistructures have been studied using the same techniques. We can cite, for example, Le Dret [1989a,1989b,1990] who modeled the junctions between plates and the junctions between beams, Ciarlet \& Miara [1992] the junction between a three-dimensional structure and a shallow shell, or Aufranc [1990] and Gruais [1991] who studied the plate-beam junction.

The stiffened plate problem in linear elasticity is seen here like a plate in which several beams are inserted; we have thus a beam-plate junction problem.

Depending on which of the two modeling technique described above is used mechanical assumptions or mathematical justification- we have two different representations in terms of partial differential equations. We will show in section 2 that in some sense the second approach justifies the first one ; from the models used in Janovsky \& Prochăska [1978] we will obtain the results of Aufranc [1990] as the thickness of the plate tends to zero for some given rigidity of the beams.

We consider here a domain decomposition without subdomain overlap, the interface being along the beams. For a conforming finite element discretisation of the Janovsky \& Prochăska's [197S] equation, the Schur complement matrix associated to the global problem is preconditioned with a specific operator associated with the beams problem only, as shown in section 3. It leads to a powerful algorithm in which the rate of convergence does not depend on the discretisation. A convergence theorem for the domain decomposition algorithm is proved and illustrated in section 4 by numerical examples.

\section{Comparison of two mathematical representa- tions of the stiffened plate problem.}

\subsection{Janovsky \& Prochăska's [1978] model}

For a given $\varepsilon$, let us consider a linearly elastic plate of thickness $\varepsilon$, in which two perpendicular beams with square cross section of diameter $\varepsilon$ are inserted. Let $E_{\varepsilon}$ and $\nu_{\varepsilon}$ be the Young modulus and the Poisson ratio of the plate and $E_{\varepsilon}^{q}$ and $\nu_{\varepsilon}^{q}$ the Young modulus and the Poisson ratio of the beams. Figure 1 shows the middle surface $S$ of the plate and the middle lines $Q_{1}$ and $Q_{2}$ of the beams in the plane $S$. We suppose that the middle lines of the beams lie on the middle surface of the plates and that they are orthogonal. The plate is clamped along $\gamma$. 


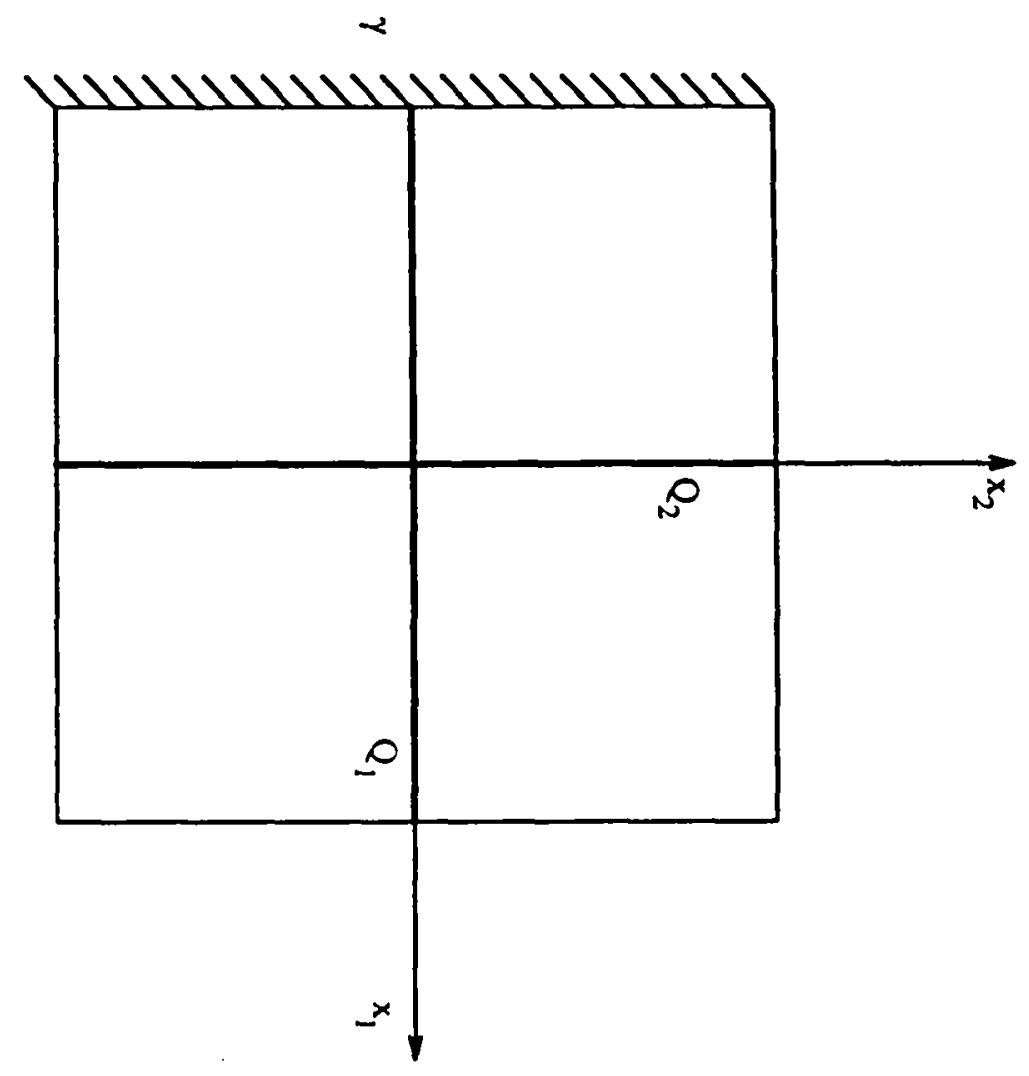

Figure 1: The middle surface of the plate and the middle lines of the beams.

$$
\begin{gathered}
S=\left\{x=\left(x_{1}, x_{2}\right) \in \mathbb{R}^{2},-1<x_{1}<1,-1<x_{2}<1\right\} \\
\gamma=\left\{x=\left(x_{1}, x_{2}\right) \in \mathbb{R}^{2}, x_{1}=-1,-1<x_{2}<1\right\} \\
Q_{1}=\left\{x=\left(x_{1}, x_{2}\right) \in \mathbb{R}^{2}, x_{2}=0,-1<x_{1}<1\right\} \\
Q_{2}=\left\{x=\left(x_{1}, x_{2}\right) \in \mathbb{R}^{2}, x_{1}=0,-1<x_{2}<1\right\} .
\end{gathered}
$$

The repeated index convention for summation is systematically used in the set $\{1,2\}$.

Let $\mathbf{V}$ be the space of admissible displacements of the stiffened plate.

$$
\begin{aligned}
& \mathbf{V}=\left\{v \in H^{2}(S), \quad v_{/ \gamma}=\left(\partial_{1} v\right)_{/ \gamma}=0, \quad v_{/ Q_{1}} \in H^{2}\left(Q_{1}\right), \quad v_{/ Q_{2}} \in H^{2}\left(Q_{2}\right),\right. \\
& \left.\left(\partial_{2} v\right)_{/ Q_{1}} \in H^{1}\left(Q_{1}\right) \text { et }\left(\partial_{1} v\right)_{/ Q_{2}} \in H^{1}\left(Q_{2}\right)\right\} \text {. }
\end{aligned}
$$

The scalar functions $v_{/ Q_{1}}$ (resp. $v_{/ Q_{2}}$ ) represent the beam flexural displacement on $Q_{1}$ (resp. $\left.Q_{2}\right)$, and the scalars functions $\left(\partial_{2} v\right)_{/ Q_{1}}\left(\right.$ resp. $\left.\left(\partial_{1} v\right)_{/ Q_{2}}\right)$ represent the torsion angle of the beam $Q_{1}$ (resp. $Q_{2}$ ).

The Janovsky \& Prochăska's [1978] stiffened plate problem for a given applied force $f \in L^{2}(S)$ has the following form : find the unique displacement $u^{\varepsilon} \in \mathbf{V}$ satis- 
fying the following variational equation

$$
\left\{\begin{aligned}
& \int_{S} \frac{E_{\varepsilon} \varepsilon^{3}}{12\left(1-\nu_{\varepsilon}^{2}\right)}\left(\left(1-\nu_{\varepsilon}\right) \partial_{\alpha \beta} u^{\varepsilon} \partial_{\alpha \beta} v+\nu_{\varepsilon} \partial_{\alpha \alpha} u^{\varepsilon} \partial_{\beta \beta} v\right) d S \\
+ & \int_{Q_{1}} I^{\varepsilon} E_{\varepsilon}^{q} \partial_{11} u^{\epsilon} \partial_{11} v d x_{1}+\int_{Q_{1}} \frac{a^{\varepsilon} E_{\varepsilon}^{q}}{2\left(\nu_{\varepsilon}^{q}+1\right)} \partial_{21} u^{\varepsilon} \partial_{21} v d x_{1} \\
+ & \int_{Q_{2}} I^{\varepsilon} E_{\varepsilon}^{q} \partial_{22} u^{\varepsilon} \partial_{22} v d x_{2}+\int_{Q_{2}} \frac{a^{\varepsilon} E_{\varepsilon}^{q}}{2\left(\nu_{\varepsilon}^{q}+1\right)} \partial_{12} u^{\varepsilon} \partial_{12} v d x_{2} \\
= & \int_{S} f v d S
\end{aligned}\right.
$$

for all $v \in \mathrm{V}$. The coefficients $I^{\varepsilon}$ and $a^{\varepsilon}$ depend on the cross section of the beams. In the case of a square cross section with diameter $\varepsilon$, we have $I^{\varepsilon}=\varepsilon^{4}$ and $a^{\varepsilon}=a \varepsilon^{4}$ with $a=9 / 64$. For more details regarding these coefficients we refer for example to Batoz \& Dhatt [1990].

Let us first give a technical lemma dealing with the fact that only one of the beams is clamped on $\gamma$.

Lemma 2.1 There exist a constant $C$ such that for all $u \in \mathrm{V}$ we have

$$
\begin{gathered}
\|u\|_{2, Q_{1}}^{2}+\|u\|_{2, Q_{2}}^{2}+\left\|\partial_{2} u\right\|_{1, Q_{1}}^{2}+\left\|\partial_{1} u\right\|_{1, Q_{2}}^{2} \\
\leq C\left(|u|_{2, Q_{1}}^{2}+|u|_{2, Q_{2}}^{2}+\left|\partial_{2} u\right|_{1, Q_{1}}^{2}+\left|\partial_{1} u\right|_{1, Q_{2}}^{2}\right) .
\end{gathered}
$$

Proof. If (2.2) is not true, there exists sequence $\left(u_{n}\right)_{n \geq 1}$ in $\mathrm{V}$ such that

$$
\left\|u_{n}\right\|_{2, Q_{1}}^{2}+\left\|u_{n}\right\|_{2, Q_{2}}^{2}+\left\|\partial_{2} u_{n}\right\|_{1, Q_{1}}^{2}+\left\|\partial_{1} u_{n}\right\|_{1, Q_{2}}^{2}=1
$$

for all $n \geq 1$, and

$$
\left|u_{n}\right|_{2, Q_{1}}^{2}+\left|u_{n}\right|_{2, Q_{2}}^{2}+\left|\partial_{2} u_{n}\right|_{1, Q_{1}}^{2}+\left|\partial_{1} u_{n}\right|_{1, Q_{2}}^{2} \underset{n \rightarrow+\infty}{\longrightarrow} 0 .
$$

The beam $Q_{1}$ being clamped on $\gamma \cap Q_{1}$, Poincaré's inequality and the above assumption allow us to write that

$$
\left\|u_{n}\right\|_{2, Q_{1}}^{2}+\left\|\partial_{2} u_{n}\right\|_{1, Q_{1}}^{2} \underset{n \rightarrow+\infty}{\longrightarrow} 0
$$

Then, in terms of trace functions, we have at the crossing point of the two beams

$$
\left\{\begin{array}{c}
u_{n}(0,0) \quad \longrightarrow 0 \\
\partial_{1} u_{n}(0,0) \longrightarrow 0 \\
\partial_{2} u_{n}(0,0) \longrightarrow 0
\end{array}\right.
$$

Using now the same arguments than in lemma 1 of Ciarlet, Le Dret \& Nzengwa [1989], as

$$
\left|u_{n}\right|_{2, Q_{2}}^{2}+\left|\partial_{1} u_{n}\right|_{1, Q_{2}}^{2} \underset{n \rightarrow+\infty}{\longrightarrow} 0
$$


then for each $n$ there exist some real numbers $a_{n}, b_{n}$ and $c_{n}$, functions $v_{n} \in H^{2}\left(Q_{2}\right)$ and $w_{n} \in H^{1}\left(Q_{2}\right)$ such that

$$
\left\{\begin{array}{l}
u_{n}\left(0, x_{2}\right)=a_{n} x_{2}+b_{n}+v_{n}\left(x_{2}\right) \\
\partial_{1} u_{n}\left(0, x_{2}\right)=c_{n}+w_{n}\left(x_{2}\right)
\end{array}\right.
$$

with $\left\|v_{n}\right\|_{H^{2}\left(Q_{2}\right)}$ and $\left\|w_{n}\right\|_{H^{1}\left(Q_{2}\right)}$ that converge to zero when $n$ tends to infinity. The continuity of the trace operator shows that

$$
v_{n}(0) \rightarrow 0, \quad \partial_{2} v_{n}(0) \rightarrow 0, \quad w_{n}(0) \rightarrow 0
$$

when $n \rightarrow+\infty$. Using (2.5), we also show that $a_{n}, b_{n}$ et $c_{n}$ are sequences with null limits. It follows that

$$
\left\|u_{n}\right\|_{2, Q_{2}}^{2}+\left\|\partial_{1} u_{n}\right\|_{1, Q_{2}}^{2} \underset{n \rightarrow+\infty}{\longrightarrow} 0 .
$$

Using (2.4) again, this leads to a contradiction with hypothesis (2.3).

We will now scale the elasticity coefficient as done in Aufranc [1990] and Gruais [1991], where two different limit problems are obtained depending on the rigidity of the beams and the plate. We will study the limit solution behavior of problem (3.1) when $\varepsilon$ tends to zero in these two cases.

\subsection{Beams with high rigidity.}

Let $E^{q}, E, \nu^{q}$ and $\nu$ be some constants independent of $\varepsilon$. In the case of high rigidity beams, as in Aufranc [1990] and Gruais [1991], we suppose that

$$
\begin{array}{ll}
E_{\varepsilon}^{q}=\varepsilon^{-i} E^{q} & \nu_{\varepsilon}^{q}=\nu^{q} \\
E_{\varepsilon}=\varepsilon^{-3} E & \nu_{\varepsilon}=\nu .
\end{array}
$$

Using the values $I^{\varepsilon}=\varepsilon^{4}$ and $a^{\varepsilon}=\varepsilon^{4} a$ as described in the previous section, equation (2.1) can be rewritten : find a unique $u^{\epsilon} \in \mathrm{V}$ that solves the following variational equation

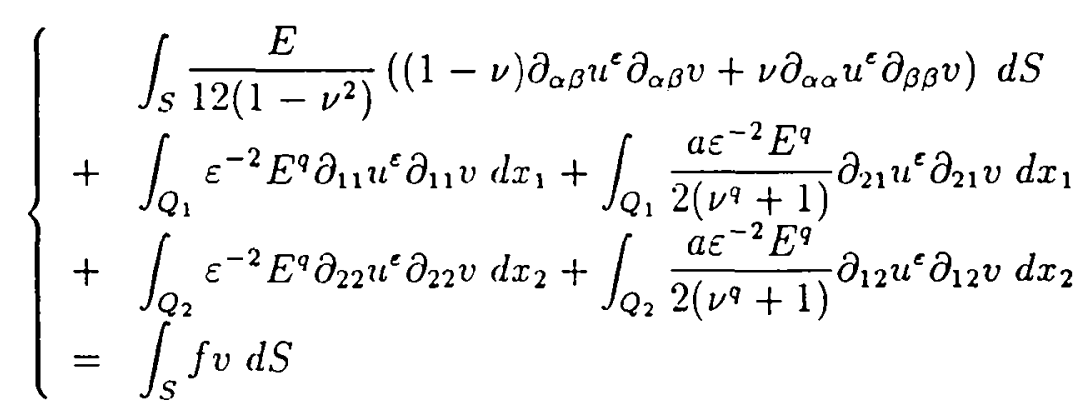

for all $v \in \mathbf{V}$.

The next lemma gives the limit problem as $\varepsilon \rightarrow 0$. 
Lemma 2.2 Let

$$
\mathbf{V}^{0}=\left\{\begin{array}{l}
v \in H^{2}(S), \quad v_{/ \gamma}=\left(\partial_{1} v\right)_{/ \gamma}=0, \quad v_{/ Q_{1}}=\left(\partial_{2} v\right)_{/ Q_{1}}=0, \\
\left.v / Q_{2}=\left(\partial_{1} v\right)_{/ Q_{2}}=0\right\} .
\end{array}\right.
$$

The sequence of $\left(u^{e}\right)_{e>0}$, solutions of (2.7), converges strongly in $\mathbf{V}$ towards a limit $u \in \mathrm{V}^{0}$ when $\varepsilon \rightarrow 0$. In addition $u$ is the unique solution in $\mathbf{V}^{\mathbf{0}}$ of the following variational problem

$$
\int_{S} \frac{E}{12\left(1-\nu^{2}\right)}\left((1-\nu) \partial_{\alpha \beta} u \partial_{\alpha \beta} v+\nu \partial_{\alpha \alpha} u \partial_{\beta \beta} v\right) d S=\int_{S} f v d S
$$

for all $v \in \mathbf{V}^{\mathbf{0}}$.

Proof. The coefficients $E, E^{q}, \nu, \nu^{q}$ and $u$ in equation (2.7) do not depend on $\varepsilon$. Then, using the boundary conditions on $\gamma$, Poincarés inequality and lemma 2.1 , there exists a constant $C$ such that for all $\varepsilon>0$

$$
\left\|u^{\varepsilon}\right\|_{2, S}^{2}+\varepsilon^{-2}\left(\left\|u^{\varepsilon}\right\|_{2, Q_{1}}^{2}+\left\|u^{\varepsilon}\right\|_{2, Q_{2}}^{2}+\left\|\partial_{2} u^{\varepsilon}\right\|_{1, Q_{1}}^{2}+\left\|\partial_{1} u^{\varepsilon}\right\|_{1, Q_{2}}^{2}\right) \leq C\|f\|_{0, S}\left\|u^{\varepsilon}\right\|_{0, S} .
$$

It follows that

$$
\left\|u^{\varepsilon}\right\|_{2, S}+\varepsilon^{-2}\left(\left\|u^{e}\right\|_{2, Q_{1}}+\left\|u^{\varepsilon}\right\|_{2, Q_{2}}+\left\|\partial_{2} u^{e}\right\|_{1, Q_{1}}+\left\|\partial_{1} u^{e}\right\|_{1, Q_{2}}\right) \leq C\|f\|_{0, s} .
$$

Consequently we have a subsequence noted $\left(u^{\varepsilon^{\prime}}\right)_{\epsilon^{\prime}>0}$ which converges weakly in $\mathrm{V}$ to a limit $u$. In addition, since

$$
\left\|u^{e}\right\|_{2, Q_{1}}+\left\|u^{e}\right\|_{2, Q_{2}}+\left\|\partial_{2} u^{e}\right\|_{1, Q_{1}}+\left\|\partial_{1} u^{e}\right\|_{1, Q_{2}} \leq \varepsilon^{2} C
$$

we show that $u$ belongs to $\mathbf{V}^{\mathbf{0}}$. To show that $u$ is the solution of the variational problem (2.8), it is sufficient to take a trial function $v \in \mathbf{V}^{\mathbf{0}} \subset \mathbf{V}$ in variational equation (2.7) and to use the weak convergence of the sequence $\left(u^{\varepsilon^{\prime}}\right)_{\varepsilon^{\prime}>0}$ to $u$. Problem (2.8) has a unique solution, thus all the sequence $\left(u^{\varepsilon}\right)_{e>0}$ converges.

Finally, to get the strong convergence of $u^{\varepsilon}$ to $u$ in $\mathrm{V}$, we use the same idea than in Ciarlet, Le Dret \& Nzengwa [19S9]. We will show that

$$
\left\{\begin{array}{l}
\theta(\varepsilon)= \\
\int_{S} \frac{E}{12\left(1-\nu^{2}\right)}\left((1-\nu) \partial_{\alpha \beta}\left(u^{\varepsilon}-u\right) \partial_{\alpha \beta}\left(u^{\varepsilon}-u\right)+\nu \partial_{\alpha \alpha}\left(u^{\varepsilon}-u\right) \partial_{\beta \beta}\left(u^{\varepsilon}-u\right)\right) d S \\
+\int_{Q_{1}} \varepsilon^{-2} E^{q} \partial_{11} u^{\varepsilon} \partial_{11} u^{\varepsilon} d x_{1}+\int_{Q_{1}} \frac{a \varepsilon^{-2} E^{q}}{2\left(\nu^{q}+1\right)} \partial_{21} u^{e} \partial_{21} u^{e} d x_{1} \\
+\int_{Q_{2}} \varepsilon^{-2} E^{q} \partial_{22} u^{e} \partial_{22} u^{\varepsilon} d x_{2}+\int_{Q_{2}} \frac{a \varepsilon^{-2} E^{q}}{2\left(\nu^{q}+1\right)} \partial_{12} u^{e} \partial_{12} u^{\varepsilon} d x_{2}
\end{array}\right.
$$

converges to zero, which is a sharper result than the convergence in $\mathrm{V}$. We write

$$
\left\{\begin{aligned}
\theta(\varepsilon)= & \int_{S} \frac{E}{12\left(1-\nu^{2}\right)}\left((1-\nu) \partial_{\alpha \beta} u^{\varepsilon} \partial_{\alpha \beta} u^{\varepsilon}+\nu \partial_{\alpha \alpha} u^{\varepsilon} \partial_{\beta \beta} u^{\varepsilon}\right) d S \\
& +\int_{S} \frac{E}{12\left(1-\nu^{2}\right)}\left((1-\nu) \partial_{\alpha \beta} u \partial_{\alpha \beta}\left(u-2 u^{\varepsilon}\right)+\nu \partial_{\alpha \alpha} u \partial_{\beta \beta}\left(u-2 u^{\varepsilon}\right)\right) d S \\
& +\int_{Q_{1}} \varepsilon^{-2} E^{q} \partial_{11} u^{\varepsilon} \partial_{11} u^{\varepsilon} d x_{1}+\int_{Q_{1}} \frac{a \varepsilon^{-2} E^{q}}{2\left(\nu^{q}+1\right)} \partial_{21} u^{\varepsilon} \partial_{21} u^{\varepsilon} d x_{1} \\
& +\int_{Q_{2}} \varepsilon^{-2} E^{q} \partial_{22} u^{\varepsilon} \partial_{22} u^{\varepsilon} d x_{2}+\int_{Q_{2}} \frac{a \varepsilon^{-2} E^{q}}{2\left(\nu^{q}+1\right)} \partial_{12} u^{\varepsilon} \partial_{12} u^{\varepsilon} d x_{2} .
\end{aligned}\right.
$$


Using the fact that $u^{e}$ is solution of (2.7) we have

$$
\left\{\begin{aligned}
\theta(\varepsilon)= & \int_{S} \frac{E}{12\left(1-\nu^{2}\right)}\left((1-\nu) \partial_{\alpha \beta} u \partial_{\alpha \beta}\left(u-2 u^{\epsilon}\right)+\nu \partial_{\alpha \alpha} u \partial_{\beta \beta}\left(u-2 u^{\varepsilon}\right)\right) d S \\
& +\int_{S} f u^{\varepsilon} d S .
\end{aligned}\right.
$$

The weak convergence of the sequence $\left(u^{\varepsilon}\right)_{e>0}$ to $u$ and equation (2.8) show that

$$
\begin{aligned}
\theta(\varepsilon) & =\int_{S} \frac{E}{12\left(1-\nu^{2}\right)}\left((1-\nu) \partial_{\alpha \beta} u \partial_{\alpha \beta}\left(u-2 u^{\varepsilon}\right)+\nu \partial_{\alpha \alpha} u \partial_{\beta \beta}\left(u-2 u^{\varepsilon}\right)\right) d S \\
& +\int_{S} f u^{\varepsilon} d S \longrightarrow 0
\end{aligned}
$$

as $\varepsilon \rightarrow 0$.

Remark. In Aufranc [1990], the mathematical modeling of the stiffened plate is a limit problem obtain from the three-dimensional linearized elasticity formulation when $\varepsilon \rightarrow 0$. With the same elasticity coefficients as in (2.6), the limit problem is a two dimensional problem identical to variational problem (2.8). Hence, we showed that Janovsky \& Prochăska's [1978] two dimensional formulation has the same asymptotic behavior than the three-dimensional elasticity model.

\subsection{Beams with high rigidity}

In this section we make the following hypothesis :

$$
\begin{array}{ll}
E_{\varepsilon}^{q}=\varepsilon^{-4} E^{q} & \nu_{\varepsilon}^{q}=\nu^{q} \\
E_{\varepsilon}=\varepsilon^{-3} E & \nu_{\varepsilon}=\nu
\end{array}
$$

with $E^{q}, E, \nu^{q}$ and $\nu$ constants independent of $\varepsilon$. In that case, problem (2.1) becomes: find the unique solution $u^{e}$ of the variational problem

$$
\left\{\begin{aligned}
& \int_{S} \frac{E}{12\left(1-\nu^{2}\right)}\left((1-\nu) \partial_{\alpha \beta} u^{\varepsilon} \partial_{\alpha \beta} v+\nu \partial_{\alpha \alpha} u^{\varepsilon} \partial_{\beta \beta} v\right) d S \\
+ & \int_{Q_{1}} E^{q} \partial_{11} u^{\varepsilon} \partial_{11} v d x_{1}+\int_{Q_{1}} \frac{a E^{q}}{2\left(\nu^{q}+1\right)} \partial_{21} u^{\varepsilon} \partial_{21} v d x_{1} \\
+ & \int_{Q_{2}} E^{q} \partial_{22} u^{\varepsilon} \partial_{22} v d x_{2}+\int_{Q_{2}} \frac{a E^{q}}{2\left(\nu^{q}+1\right)} \partial_{12} u^{e} \partial_{12} v d x_{2} \\
= & \int_{S}^{f} f v d S .
\end{aligned}\right.
$$

for all $v \in \mathbf{V}$.

The coefficients of this equation do not depend on $\varepsilon$ any longer. Thus, its solution does not depend on $\varepsilon$.

In Gruais [1991], the mathematical modeling of the plate-beam junction is a limit problem obtain from the three-dimensional linearized elasticity system when $\varepsilon \rightarrow 0$. The solution for this model, with the same elasticity coefficients as in (2.9), has 
a torsional displacement of the beam in the plate that remains constant along the junction.

This is not the case in Janovsky \& Prochăska's [1978] model given by equation (2.10), using the elasticity coefficients like in (2.9); one can see that the torsion energy of the beam is finite. Consequently, it appears that these models do not have the same asymptotic behavior when the thickness tends to zero.

Then, we proved that equations (2.1) cannot represent a beam-plate junction with a low rigidity of the beam.

Nevertheless, from the numerical point of view, Gruais's [1991] problem might be seen like Janovsky \& Prochăska's [1978] one with an infinite torsion energy. Thus, the domain decomposition algorithm we present below will remain valid for the both models.

\section{A specific domain decomposition method for stiffened plates}

Consider a decomposition of $S$ with non-overlaping subdomains where the interfaces are along the beams. We will take advantage of the energy terms in the variational equation for the displacement along $Q_{1}$ and $Q_{2}$.

The method we will present could be formulated in continuous spaces. However, it is more convenient to discretize the equation first and then, to apply the method in a finite dimensional space. The numerical analysis of the method consists in showing that the convergence only depends on the nature of the problem and not on its discretisation.

\subsection{Conforming finite element discretisation}

Let $\mathcal{T}_{h}$ be a mesh on $S$ where $h$ denotes the diameter of the elements. We suppose that $Q_{1}$ and $Q_{2}$ coincide with edges of the mesh. Let $V^{h}$ be the discrete space resulting from the discretisation. We use H-C-T $\mathcal{C}^{1}$-elements (see Ciarlet [1978]), which are piecewise polynomials of degree three on each triangle of $\mathcal{T}_{h}$.

Along the edges of these elements, the unknown is a polynomial of degree three, and the normal derivative of the unknown is of degree two. In this way, on each edge of an element of $\mathcal{T}_{h}$ lying on $Q_{1}$ or $Q_{2}$, the flexural displacement of the beams, discretized by polynomials of degree three will be equal to the flexural displacement of the plate, and the angle of torsion of the beams discretized with polynomials of degree two will be equal to the normal derivative of the flexural displacement of the plate. Figure 2 represents the coupling between the seven degrees of freedom of an H.C.T. element along $Q_{1}$ and $Q_{2}$, with on the one hand, the four degrees of freedom for the beam's flexural displacement - value at the nodes and tangential derivative at the vertices - and, on the other hand, the three degrees of freedom for the beam's rotation - normal derivative at the vertices and in the middle of the edge.

Let $Q=Q_{1} \cup Q_{2}$ be the interface between subdomains $\left(S_{i}\right)_{i=1}^{4}$ of $S$ (see fig. 3 ). In 
other words, we have

$$
\cup_{i=1}^{4} \bar{S}_{i}=\bar{S} \quad \text { and } \quad \cap_{i=1}^{4} \bar{S}_{i}=Q
$$

Let $t r^{Q}$ be the trace operator along $Q$ associating to every function $v \in \mathbf{V}^{h}$

$$
\operatorname{tr}^{Q} v=\left(v_{/ Q},\left(\partial_{\nu} v\right)_{/ Q}\right)
$$

where $\nu$ is the unit vector normal to $Q$ (see figure 3 ). Then

$$
V_{Q}=\left\{\left(v_{/ Q},\left(\partial_{\nu} v\right)_{/ Q}\right)=\operatorname{tr}^{Q} v, v \in \mathbf{V}^{h}\right\}
$$

is the space of discrete trace functions of $V^{h}$ on $Q$.

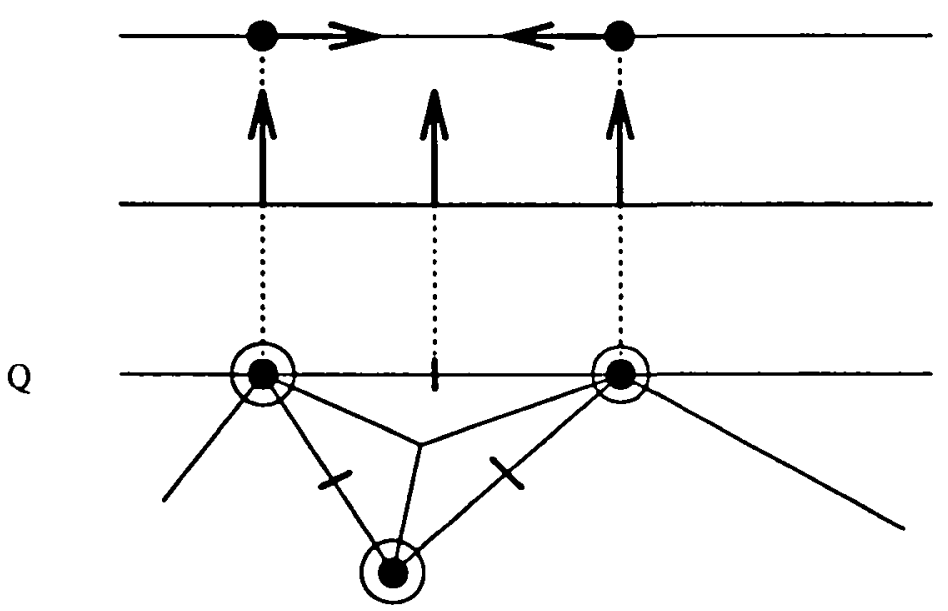

Beam flexion element P3

Beam torsion element P2

Plate element H.C.T.

Figure 2: Coupling of a H.C.T. 2-D finite element for plates with a 1-D finite element for the flexural and torsional displacement of the beam.

After the discretisation, for a given $h$, problem (2.1) becomes : find the unique solution $u_{h} \in \mathrm{V}^{h}$ of the variational problem

$$
a^{p}\left(u_{h}, v_{h}\right)+a^{q}\left(t r^{Q} u_{h}, t r^{Q} v_{h}\right)=\int_{S} f v_{h} d S \text { for all } v_{h} \in \mathbf{V}^{h}
$$

where

$$
a^{p}\left(u_{h}, v_{h}\right)=\int_{S} \frac{\varepsilon^{3} E_{\varepsilon}}{12\left(1-\nu_{\varepsilon}^{2}\right)}\left(\left(1-\nu_{\varepsilon}\right) \partial_{\alpha \beta} u_{h} \partial_{\alpha \beta} v_{h}+\nu_{\varepsilon} \partial_{\alpha \alpha} u_{h} \partial_{\beta \beta} v_{h}\right) d S
$$

is the variational term for the flexural displacement of the plate, and

$$
\begin{aligned}
a^{q}\left(t^{Q} u_{h}, t r^{Q} v_{h}\right)= & \int_{Q_{1}} I^{\varepsilon} E_{\varepsilon}^{q} \partial_{11} u_{h} \partial_{11} v_{h} d x_{1}+\int_{Q_{1}} \frac{a^{\varepsilon} E_{\varepsilon}^{q}}{2\left(\nu_{\varepsilon}^{q}+1\right)} \partial_{21} u_{h} \partial_{21} v_{h} d x_{1} \\
& +\int_{Q_{2}} I^{\varepsilon} E_{\varepsilon}^{q} \partial_{22} u_{h} \partial_{22} v_{h} d x_{2}+\int_{Q_{2}} \frac{a^{\varepsilon} E_{\varepsilon}^{q}}{2\left(\nu_{\varepsilon}^{q}+1\right)} \partial_{12} u_{h} \partial_{12} v_{h} d x_{2},
\end{aligned}
$$

is the variational term for the flexural and torsional displacement of the beams. 


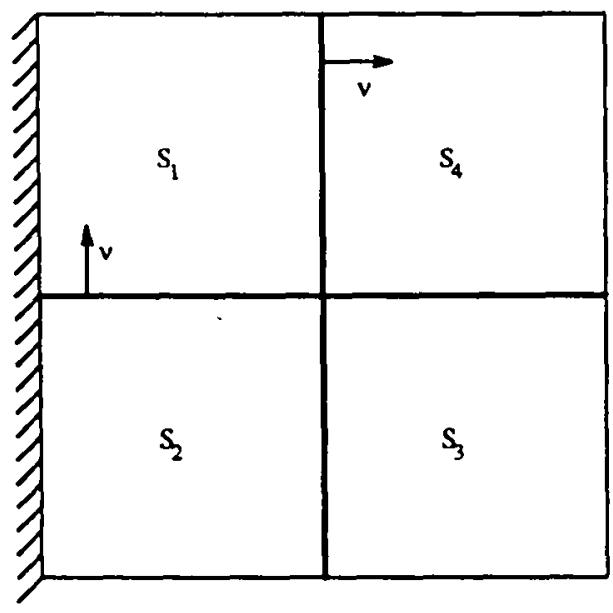

Figure 3: Decomposition of the plate in four subdomains along the beams.

We now define the following discrete spaces

$$
\mathrm{V}_{i}=\left\{w=v_{/ s_{i}}, v \in \mathrm{V}^{h}\right\}
$$

and

$$
\mathbf{V}_{i}^{0}=\left\{v \in V_{i}, \text { with } \operatorname{tr}^{Q \cap a S_{i}} v=0\right\}
$$

The solution of problem (3.1) does not have enough regularity to allow for a good error approximation with a classical technique (in the sense of Ciarlet[1978]). This problem has been solved in Janovsky \& Prochăska [1978] : the solution of the continuous problem is approximated with an arbitrary accuracy in the energy norm by a function having sufficient regularity. Their paper discusses a non conforming finite element : the same ideas may be applied to H.C.T. finite elements.

\subsection{Toward an interface problem}

In many domain decomposition methods, the global problem is transformed into a problem posed on the interface between subdomains. Here we introduce the functions $\left(u_{i}^{I}\right)_{i=1}^{4}$ which are solutions of the variational problems

$$
\left\{\begin{array}{l}
u_{i}^{I} \in V_{i}^{0} \\
a^{p}\left(u_{i}^{I}, v\right)=\int_{S_{i}} f v d x, \quad \forall v \in V_{i}^{0} .
\end{array}\right.
$$

From the definition of $u_{i}^{I}$, for any given $v$ in $\mathrm{V}^{h}$, the real number

$$
\sum_{i=1}^{4}\left[a^{p}\left(u_{i}^{I}, v\right)-\int_{S_{i}} f v d S\right]
$$

depends only on $t r^{Q} v$. Thus, we can define a linear form on $V_{Q}$, which maps every $v \in \mathbf{V}^{h}$ onto

$$
<b, t r^{Q} v>_{Q}=\sum_{i=1}^{4}\left[a^{p}\left(u_{i}^{l}, v\right)-\int_{S_{i}} f v d S\right]
$$


where $<, . .>_{Q}$ denotes the dual product between $V_{Q}$ and $V_{Q}^{*}$. Let $u^{I}$ be the function such that $u_{/ S_{i}}^{I}=u_{i}^{I}$.

In order to obtain the solution of (3.1), it remains to compute $u_{i}^{H} \in V_{i}$ so that $u_{i}^{I}+u_{i}^{H}$ is the solution restricted to $S_{i}$. Then, if we denote $u^{H}$ to be the function equal to $u_{i}^{H}$ on $S_{i}$, it is sufficient that

$$
\left\{\begin{array}{l}
u^{H} \in \mathbf{V}^{h} \\
\sum_{i=1}^{4} a^{p}\left(u_{i}^{H}, v\right)+a^{q}\left(t^{Q} u^{H}, t r^{Q} v\right)=-<b, t r^{Q} v>\quad \text { for all } v \in \mathbf{V}^{h}
\end{array}\right.
$$

Indeed, using the definitions of $u^{I}$ and $b$, we have

$$
\left\{\begin{array}{l}
u^{H}+u^{I} \in \mathbf{V}^{h} \\
\sum_{i=1}^{4} a^{p}\left(u_{i}^{H}, v\right)+a^{q}\left(t r^{Q} u^{H}, t r^{Q} v\right)=-\left[a^{p}\left(u^{I}, v\right)-\int_{S} f v d S\right] \quad \text { for all } v \in \mathbf{V}^{h}
\end{array}\right.
$$

which in nothing but problem (3.1). Since $b$ is a linear form on $V_{Q}$, problem (3.3) can be seen as an interface problem.

\subsection{The Schur matrix for the stiffened plate problem}

In this section we will make no distinction between operators defined on finite dimensional spaces and the corresponding matrices in $\mathbb{R}^{N}$ with $N$ equal to the dimension of the finite dimensional spaces.

For $r \in V_{Q}$, we denote $u_{i}^{r}, i=1, \ldots, 4$, to be the unique solution of the variational problem

$$
\left\{\begin{array}{l}
u_{i}^{r} \in V_{i} \quad t r^{Q \cap \partial S_{i}} u_{i}^{r}=r \\
a^{p}\left(u_{i}^{r}, v\right)=0 \quad \forall v \in V_{i}^{0} .
\end{array}\right.
$$

In the following $<., .>_{Q}$ will denote the duality product between $V_{Q}^{*}$ and $V_{Q}$. Let $q \in V_{Q}^{*}$ be the linear form defined as follows

$$
<q, t^{Q} v>_{Q}=\sum_{i=1}^{4} a^{p}\left(u_{i}^{r}, v\right)+a^{q}\left(r, t r^{Q} v\right) \quad \forall v \in \mathbf{V}^{h}
$$

Indeed, from the definition of $\left(u_{i}^{r}\right)_{i=1}^{4}$, the right-hand-side only depends on $t r^{Q} v$. Using these notations we can define the interface operator

$$
T:\left\{\begin{array}{ccc}
V_{Q} & \longrightarrow V_{Q}^{*} \\
r & \longmapsto q
\end{array}\right.
$$

which is nothing but the Schur complement matrix (see for example Bjorstad \& Widlund [1986]) resulting from a block Gauss factorization of the global matrix of the discretized problem.

The inequality shown in the next lemma is specific to the stiffened plate problem. 
Lemma 3.1 The operator $T$ defined in (3.6) is symmetric and positive definite. In addition, there exists a constant $C$ independent of $h$ such that for all $r \in V_{Q}$,

$$
<T r, r>_{Q} \leq C a^{q}(r, r)
$$

Proof. Since the bilinear form

$$
(u, v) \mapsto a^{p}(u, v)+a^{q}\left(t r^{Q} u, t r^{Q} v\right)
$$

is symmetric and positive definite, and using the definition (3.6), one can see that $T$ is also symmetric and positve definite.

To prove (3.7) it is sufficient to show that for all $r \in V_{Q}$, the solution $u_{i}^{r}$ of (3.4) satisfies the a priori estimate

$$
a^{p}\left(u_{i}^{r}, u_{i}^{r}\right) \leq C_{i}\left(\left\|u_{i}^{r}\right\|_{\frac{3}{2}, Q \cap \partial S_{1}}^{2}+\left\|\partial_{\nu} u_{i}^{r}\right\|_{\frac{1}{2}, Q \cap \partial S_{1}}^{2}\right)
$$

with $C_{i}$ independent of $h$. Indeed, if $u^{r} \in V^{h}$ denotes the function equal to $u_{i}^{r}$ on $S_{i}$, the continuous Sobolev embedding properties allow us to write

$$
\begin{aligned}
a^{p}\left(u^{r}, u^{r}\right)=\sum_{i=1}^{4} a^{p}\left(u_{i}^{r}, u_{i}^{r}\right) & \leq \max _{i=1, \ldots, 4} C_{i} \sum_{i=1}^{4}\left(\left\|u_{i}^{r}\right\|_{\frac{3}{2}, Q \cap \partial S_{i}}^{2}+\left\|\partial_{\nu} u_{i}^{r}\right\|_{\frac{1}{2}, Q \cap \partial S_{i}}^{2}\right) \\
& \leq C \sum_{i=1}^{4}\left(\left\|u_{i}^{r}\right\|_{2, Q \cap \partial S_{i}}^{2}+\left\|\partial_{\nu} u_{i}^{r}\right\|_{1, Q \cap \partial S_{1}}^{2}\right) \\
& \leq C\left(\left\|u^{r}\right\|_{2, Q}^{2}+\left\|\partial_{\nu} u^{r}\right\|_{1, Q}^{2}\right) .
\end{aligned}
$$

Then it remains to use lemma 2.1 which proves the coercivity of the bilinear form

$$
(r, s) \mapsto a^{g}(r, s)
$$

in $H^{2}(Q) \times H^{1}(Q)$ to get the result.

In order to prove (3.8), let us define $w_{i}^{r}$, the solution of the continuous problem

$$
\left\{\begin{array}{l}
w_{i}^{r} \in H^{2}\left(S_{i}\right), \quad \operatorname{tr}^{Q \cap \partial S_{i}} w_{i}^{r}=r \\
a^{p}\left(w_{i}^{r}, v\right)=0, \quad \forall v \in H^{2}\left(S_{i}\right) \text { with } t r^{Q \cap \partial S_{i}} v=0,
\end{array}\right.
$$

with $r \in V_{Q}$. For subdomains with shapes given here, there always exist an $\alpha>0$ such that $w_{i}$ belongs to $H^{2+\alpha}\left(S_{i}\right)$ (see for example Blum \& Rannacher [1980]). Thus, the interpolation operator associated to the finite element discretisation, using the first derivative as degree of freedom is well defined on $w_{i}$, since $H^{2+\alpha}\left(S_{i}\right) \subset \mathcal{C}^{1}\left(S_{i}\right)$ for any $\alpha>0$ (see Ciarlet [1978]). The rest of the proof uses the same ideas than Bjorstad \& Widlund [1986] or Bramble, Pasciak \& Schatz [1986]. We can write

$$
a^{p}\left(u_{i}^{r}, u_{i}^{r}\right)^{1 / 2} \leq C\left\|u_{i}^{r}\right\|_{2, S_{1}} \leq C\left(\left\|u_{i}^{r}-w_{i}^{r}\right\|_{2, S_{1}}+\left\|w_{i}^{r}\right\|_{2, S_{i}}\right) .
$$

Using an error estimate for finite element methods (cf. Ciarlet [1978, p. 104]) we have

$$
\left\|w_{i}^{r}-u_{i}^{r}\right\|_{2, S_{i}} \leq C\left\|w_{i}^{r}-\Pi_{h} w_{i}^{r}\right\|_{2, S_{i}},
$$


whe $\Pi_{h}$ denotes the interpolation operator for the H.C.T. finite element. An interpolation result in fractional order Sobolev spaces shown in Sanchez \& Arcangéli [1984], allows us to write

$$
\left\|w_{i}^{\tau}-\Pi_{h} w_{i}^{\tau}\right\|_{2, S_{i}} \leq C h^{\alpha}\left\|w_{i}^{\tau}\right\|_{2+\alpha, S_{i}} .
$$

As $w_{i}^{r}$ is the solution of a continuous problem, the following a priori estimate with a constant $C$ independent of $h$ holds:

$$
\left\|w_{i}^{r}\right\|_{2+\alpha, S_{i}} \leq C\left(\left\|u_{i}^{\tau}\right\|_{\frac{3}{2}+\alpha, \partial S_{i} \cap Q}^{2}+\left\|\partial_{\nu} u_{i}^{\tau}\right\|_{\frac{1}{2}+\alpha, \partial S_{i} \cap Q}^{2}\right) .
$$

Due to an inverse inequality on the discrete trace functions $u_{i}^{r} / \partial S_{1} \cap Q$ and $\left(\partial_{\nu} u_{i}^{r}\right) / \partial S_{1} \cap Q$ (see Babuška \& Aziz [1972], or Ciarlet [1978, p. 140]) we have

$$
h^{\alpha}\left\|w_{i}^{r}\right\|_{2+\alpha, S_{1}} \leq C\left(\left\|u_{i}^{\tau}\right\|_{\frac{3}{2}, \partial S, \cap Q}^{2}+\left\|\partial_{\nu} u_{i}^{r}\right\|_{\frac{1}{2}, \partial S, \cap Q}^{2}\right) .
$$

Using again an a priori estimate for the continuous solution, we also have

$$
\left\|w_{i}^{r}\right\|_{2, S,} \leq C\left(\left\|u_{i}^{r}\right\|_{\frac{3}{2}, \partial S, \cap Q}^{2}+\left\|\partial_{\nu} u_{i}^{r}\right\|_{\frac{1}{2}, \partial S, \cap Q}^{2}\right) .
$$

By substitution of these estimates in (3.9), we finally obtain the estimate (3.8).

\subsection{A preconditioner for the interface problem}

Using definition (3.6) of $T$, the interface problem (3.3) can be written in the following manner :

$$
\text { Find } r \in V_{Q} \text { such that } T r=-b \text { in } V_{Q}^{*} \text {. }
$$

The solution $u^{H}$ of the interface problem (3.3) will then be equal to the solution $u_{i}^{r}$ of (3.4) depending on $r \in V_{Q}$ solution of (3.12).

In order to solve (3.12), we will use the conjugate gradient method. For details about this method, consult Ciarlet [19S2] or Lascaux \& Théodor [1986] for a description of convergence estimates. It is shown that the number of iterations to reach a given accuracy depends on the conditioning of the operator to be inverted. In the case of the stiffened plate problem, it is possible to transform problem (3.12) in such a way as to have a small condition number at a very small computational cost.

In other words we introduce a preconditioner. Let $R$ be the operator :

$$
R:\left\{\begin{array}{ccc}
V_{Q} & \longrightarrow & V_{Q}^{*} \\
s & \mapsto & \chi
\end{array}\right.
$$

with $\chi$ the unique element in $I_{\ddot{q}}$ such that

$$
a^{i}(s, t)=<\chi, t>_{Q} \quad \forall t \in V_{O} .
$$


Since the bilinear form $(s, t) \longrightarrow a^{q}(s, t)$ is symmetric and positive definite (see lemma 2.1 ), operator $R$ is symmetric and positive definite. It follows that problem (3.12) can be written equivalently

$$
\left\{\begin{array}{l}
\text { Find } r \in V_{Q} \text { such that } \\
R^{-1} T r=-R^{-1} b \text { in } V_{Q} .
\end{array}\right.
$$

For any $r$ and $s$ in $V_{Q}$, let us denote

$$
[r, s]_{Q}=<R r, s>_{Q}
$$

to be the scalar product associated with operator $R$; then

$$
\left[R^{-1} T r, s\right]_{Q}=<\operatorname{Tr}, s>_{Q} \text {. }
$$

Hence, the operator $R^{-1} T$ is symmetric and positive definite for the scalar product $[.,]_{Q}$. The conjugate gradient method can be applied to (3.14) with respect to the scalar product $[., .]_{Q}$. This allows us to show an estimate for the conditioning of $R^{-1} T$ in the next theorem that justifies the convergence of the method (see Lascaux \& Théodor [1986]).

Theorem 3.1 There cxist a positive constant $C$, not depending on $h$, such that for all $r \in V_{Q}$,

$$
[r, r]_{Q} \leq\left[R^{-1} T r, r\right]_{Q} \leq C[r, r]_{Q}
$$

Proof. We have

$$
[r, r]_{Q}=<R r, r>_{Q}=a^{q}(r, r) \leq a^{q}(r, r)+\sum_{i=1}^{4} a^{P}\left(u_{i}^{r}, u_{i}^{r}\right)=<T r, r>_{Q}=\left[R^{-1} T r, r\right]_{Q} .
$$

Using lemme 4.1

$$
\left[R^{-1} T r, r\right]_{Q}=<T r, r>_{Q} \leq C a^{q}(r, r)=C<R r, r>_{Q}=C[r, r]_{Q},
$$

with $C$ independent of $h$.

\section{Remarks.}

(i) The preconditioner introduced here has the advantage of solving the interface problem (3.12) by a number of iterations which do not depend on the discretisation, without expensive calculations. Indeed, the preconditioner is an elliptic operator on a one-dimensional space ; then, in each iteration the cost for the computation of $R^{-1} \chi$, for a given $\chi \in V_{Q}^{*}$ (see problem (3.13)), is small compared to the plate problem (3.4) on each subdomain for the computation of $\operatorname{Tr}$.

(ii) We have considered here the case of two crossed beams. A real-life case may present a large number of beams. That would not present a handicap in our method, since the convergence properties will not be modified. In other words, the conditioning of problem (3.14) remains the same, if on a given plate, one adds several beams with corresponding interfaces and subdomains. Indeed, the constants $C_{i}$ that appear in the proof of lemma 3.1 do not depend on the size of subdomain $S_{i}$ (see Widlund [1988]). 


\subsection{Numerical study}

The domain decomposition method solving the interface problem (3.12) with a conjugate gradient method has been implemented within the finite element library MODULEF (see Bernadou et al. [1988]).

We will consider three tests. The first one is nothing but the case presented in the previous section with two crossing beams and four subdomains (see figure 3 ). The second one is applied with the geometry represented in figure 4 ; here again we have a square geometry clamped on one side, with six beams and sixteen subdomains. Lastly, we consider a slender structure represented in figure 5 , with eight subdomains and a clamping condition on the small side.

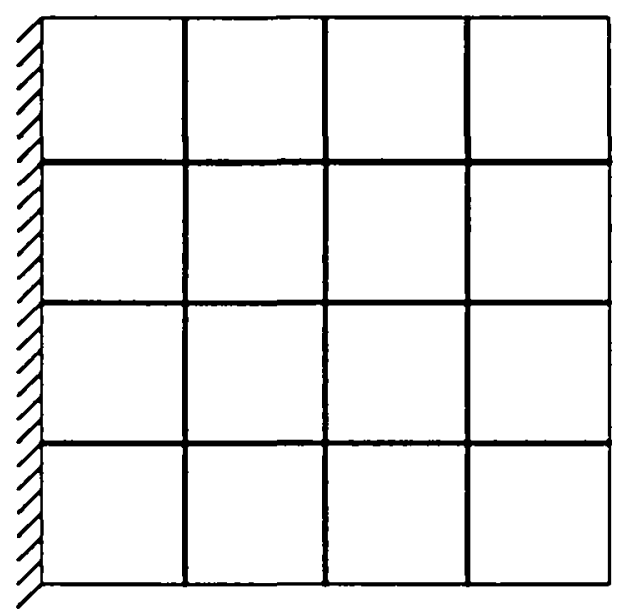

Figure 4: Plate decomposition in sixteen subdomains along the beams.

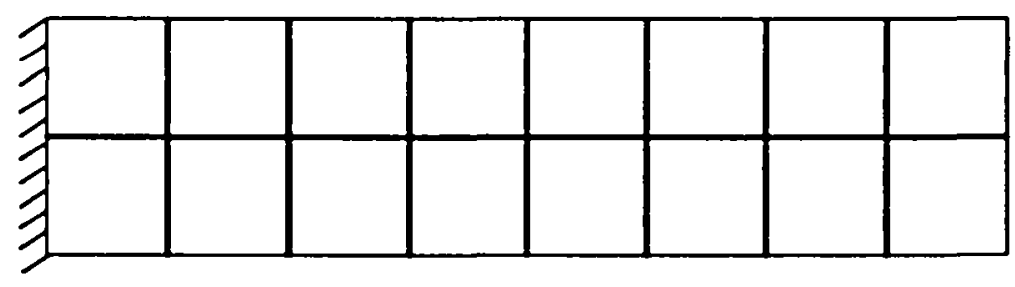

Figure 5: Stiffened slender plate.

In each case we will consider two rigidities for the beams as in section 2.1 2.3 with a given uniform load on the plate. The Poisson ratio for the plate and for the beams is $\nu=0,3$. We have $E_{q}=E$ (see the hypothesis on the materials (2.6) and $(2.9))$.

The following cases are investigated:

Test a : square plate with 2 beams, 4 subdomains and 18 finite elements in each subdomain

Test b : square plate with 2 beams, 4 subdomains and 162 finite elements in each subdomain 
Test c : square plate with 9 beams, 16 subdomains 18 finite elements in each subdomain

Test $\mathrm{d}$ : square plate with 9 beams, 16 subdomains 162 finite elements in each subdomain

Test e : slender plate with 8 beams, 16 subdomains 18 finite elements in each subdomain

Test $f$ : slender plate with $\delta$ beams, 16 subdomains 162 finite elements in each subdomain

\subsubsection{Results for high rigidity beams}

The following table gives the number of iterations necessary to reach convergence, with a relative residual lower than $10^{-6}$.

\begin{tabular}{|c|c|c|c|c|c|c|}
\hline thickness & $\mathrm{a}$ & $\mathrm{b}$ & $\mathrm{c}$ & $\mathrm{d}$ & $\mathrm{e}$ & $\mathrm{f}$ \\
\hline \hline 0.1 & 3 & 3 & 3 & 3 & 3 & 3 \\
\hline 0.01 & 2 & 2 & 2 & 2 & 2 & 2 \\
\hline 0.001 & 1 & 1 & $\mathrm{l}$ & 1 & 1 & 1 \\
\hline
\end{tabular}

\section{Table 1 : number of iterations for high rigidity beams}

It is clear that the convergence neither depends on the discretisation nor on the number of beams. This is an illustration of the results obtained in section 3.4.

We have seen in lemma 2.2, that the limit problem as $\varepsilon \rightarrow 0$ with a high rigidity of the beams, leads to independent problems on each subdomains with null flexion and torsion of the beams. This results are consistent with the numerical tests. Indeed, for a thickness equal to 0.001 , the algorithm converges after a single iteration. But the numerical solution for the flexion and torsion of the beam is practically zero in comparison with the flexion of the plate. This means that it is not necessary to use a domain decomposition method in this case ; an independent computation on every subdomain with Dirichlet boundary conditions equal to the solution of the beam problem is enough. This also means that the numerical method "sees" the disconnection between the subdomains : in this case we have an exact preconditioner.

By the contrary, for bigger thickness, we have the result of a coupled problem in two or three iterations.

\subsubsection{Results for low rigidity beams}

In this case, we consider problem (3.1) with an infinite torsion energy for the beams; from the numerical point of view, we have :

$$
\frac{a^{\varepsilon} E_{\varepsilon}^{q}}{2\left(\nu_{\varepsilon}^{q}+1\right)}=10^{+9}
$$

In that way, the torsional displacement of the beam will remain constant along $Q_{1}$ and $Q_{2}$ as in Gruais's [1991] model. We consider the same tests a. to f. than in section 
3.5.1. In the case of a low rigidity of the beams (see $\S 2.3$ ), the problem does not depend on the thickness of the plate and beams. The next table gives the number of iterations needed to reach convergence for a given thickness.

\begin{tabular}{|c|c|c|c|c|c|c|}
\hline test & a & b & c & d & e & f \\
\hline \hline iterations & 4 & 4 & 4 & 4 & 5 & 5 \\
\hline
\end{tabular}

\section{Table 2 : Number of iterations with low rigidity beams}

Here again, the result is neither dependent on the discretisation nor on the number of the beams. It illustrates the result of theorem 3.1, with an infinite torsion energy for the beams. Then we have an efficient algorithm in the case of a low beam rigidity for Gruais's [1991] model.

\section{Conclusion}

In the numerical simulation of a multistructure, it is convenient to use domain decomposition with interfaces along the junctions, in order to have on each subdomain a classical problem of three-dimensional elasticity, plate, shell, beams... The case of the plate-beam junction seen as a stiffened plate problem is, from its mechanical specificity, well suited to the application of a domain decomposition method as shown by the convergence theorem and numericals results.

The idea that has been used here, might be generalized to other multi-dimensional problems, the lower dimension part being considered as the interface - or part of the interface- between the subdomains. This idea is consistent with the fact that in the modeling of these problems, the lower dimensional part is inserted in the higher dimensional part. Consult Ciarlet, Le Dret \& Nzengwa [1989] and the first part of Aufranc [1990] on this topic.

\section{REFERENCES.}

Aufranc, M. [1990]: Sur quelques Problèmes de Jonctions dans les Multistructures Elastiques, Thèse de l'Université Pierre et Marie Curie, Paris.

BABUŠKA, I.; AZIZ A.K. [1972]: Part 1: Survey Lectures on the Mathematical Foundation of the Finite Element Method, The mathematical foundation of the finite element method with application to partial differential equations (A.K. Azız ed), Academic Press, New-York.

Batoz, J.L.; Dhatt, G. [1990]: Molélisation des Structures par Eléments Finis. Volume 2: Poutres et Plaques, Hermes, Paris.

BERnADOU, M. [19S9] : Variational formulation and approximation of junctions between thin shells, Proccedings of the fifth International Symposium on Numerical Methods in Engincering, (R. GRuber, J. Periaux, R.P. Shaw eds) I, 
pp. 407-414, Computational Mechanics, Southampton Boston, Springer-Verlag Berlin Heidelberg New-York London Paris Tokyo.

Bernadou,; M. Fayolle, S.; Lene, F. [1989]: Numerical analysis of junctions between plates, Comp. Meth. Appl. Mech. Engrg., 74, pp. 307-326.

Bernadou, M.; George, P.L.; Hassim, A.; Joly, P.; Laug, P.; Perronnet, A.; Saltel, E.; Steer, D.; VanderborCK, G.; Vidrascu, M. [1988]:

MODULEF, Une Bibliothèque modulaire d'Eléments Finis, INRIA, Rocquencourt.

BJorstad, P.E.; Widlund, O.B. [19S6]: Iterative methods for the solution of elliptic problems on regions partitioned into substructures., SIAM J. Numer. Analysis, 23, pp. 1097-1120.

Blum, H.; RanNacheR, R. [1980]: On the boundary value problem of the biharmonic operator on domains with angular corners, Math. Meth. in the Appl. Sci. 2, pp. 556-581.

Bramble, J.H.; Pasciak, .J.E.; SchatZ, A.H. [19S6]: An iterative method for elliptic problems on regions partioned into substructures, Math. Comp., 46, pp. 361-369.

Ciarlet, P.G. [1978]: The Finite Element Method for Elliptic Problems, NorthHolland.

Ciarlet, P.G. [19S2]: Introduction à l'Analyse Numérique Matricielle et à l'Optimisation, Masson, Paris.

Ciarlet, P.G.; Le Dret, H.; Nzengwa, R. [1989]: Junctions between threedimensional and two-dimensional linearly elastic structures, J. Math. Pures Appl., 68, pp. 261-296.

Ciarlet, P.G.; Miara, B. [1992]: Junctions between linearly elastic three-dimensional structures and shallow shells, to appear.

Gruais, I. [1991]: Analyse Asymptotique de quelques Problèmes de Jonction en Théorie des Plaques et des Poutres Elastiques. Thèse de l'Université Pierre et Marie Curie, Paris.

Janovsky, V.; Prochāska, P. [1976]: The nonconforming finite element method in the problem of clamped plate with ribs, Aplikace Matematiky, 21, pp. 273289.

JANOVSKY, V.; ProchăSKa, P. [1978]: Convergence analysis of a nonconforming finite element method solving a plate with ribs, Aplikace Matematiky, 23, pp. 9-29.

Lascaux, P.; Theodor, R. [1986]: Analyse Numérique Matricielle Appliquée à l'Art de l'Ingénieur, Masson, Paris, 1986. 
Le Dret, H. [19S9a]: Folded plates revisited, Comput. Mech., 5, pp. 345-365.

Le Dret, H. [1989b]: Modeling of a junction between two rods, J. Math. Pures Appl., 68, pp. 365-397.

Le Dret, H. [1990]: Modeling of a folded plate, Comput. Mech., 5, pp. 401-416.

SANCHEZ, A.M.; ARCANGÉLI R. [1984]: Estimation des erreurs de meilleure approximation polynômiale et d'interpolation de Lagrange dans les espaces de Sobolev d'ordre non entier, Numer. Math., 45, pp 301-321.

WIDLUND, O. [1988]: Iterative substructuring methods : Algorithms and theory for the elliptic problem in the plane, in the First International Symposium on Domain Decomposition Method for Partial Differential Equations, (R. GLowinSki, G.H. Golub, J.PeriauX, eds), Paris, SIAM editor, Philadelphia 1988. 

ISSN $1249-6399$ 